\title{
Always read the label carefully
}

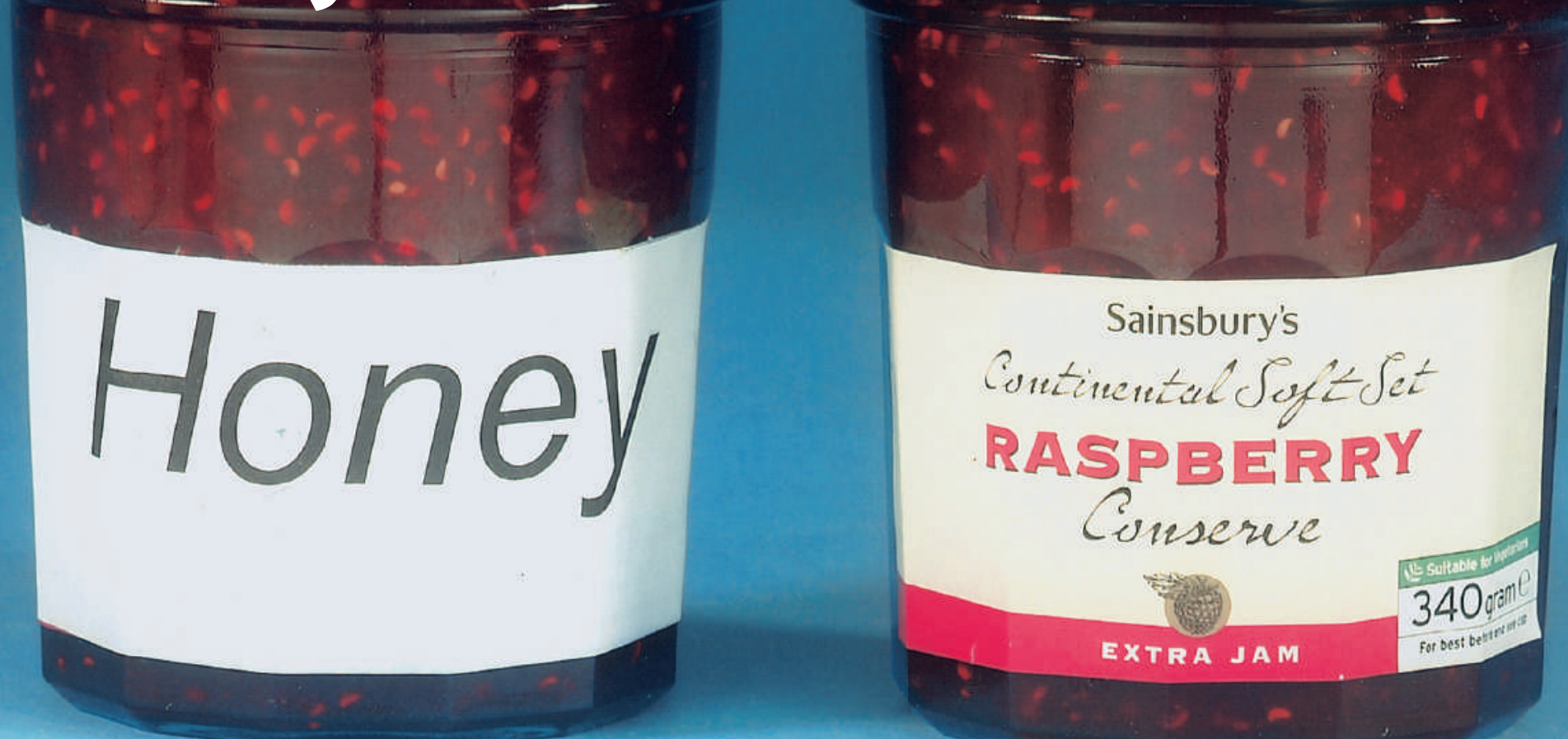

Laurence Bindoff

Institute of Neurology, Haukeland Sykehus, 5021

Bergen, Norway; E-mail: Laurence.Bindoff@nevro

.uib.no

Practical Neurology, $\mathbf{2}$, 302-303
I must admit that my first reactions on being asked to contribute to this section were negative. I felt I might be damned if I did (litigation or ridicule) and damned if I did not (something to hide). I accepted the task therefore with some apprehension.

The learning experience I would like to share concerns a common neurological condition and the issue of diagnostic labelling. The error, if that is indeed what it was, I have made more than this once and I am not sure that I will not transgress again.

The patient was 68 years old when I first saw him. He was referred because of tremor present for around 17 years, as well as involuntary movements of his right arm of more recent onset. $\mathrm{He}$ had been seen by a neurologist in another region many years previously and diagnosed as having an essential tremor. This diagnosis was later changed when he showed a response to L-dopa so when I saw him he thought he had Parkinson's disease and was still taking L-dopa.

On examination he had a mild, symmetrical positional tremor of his upper limbs, and titubation. In addition, he had choreoathetotic movements of his right arm and a mild spastic paraparesis. There was little rigidity or brady- kinesia. My first impression was that, whilst he could have well-controlled Parkinson's disease with treatment-related choreoathetosis, he was more likely to have some other neurodegenerative disease. When subsequently I learned from one of his children that the patient's father and two paternal siblings had had neurological disorders, including one with late-onset parkinsonism and one with ataxia, my suspicions appeared confirmed.

During a later consultation I discussed the question of diagnosis with the patient and told him that that I did not think he had Parkinson's disease. I said that he had another disorder causing similar symptoms, one that could affect other parts of the brain and be inherited. I suggested that we should do some more tests to investigate these possibilities, including genetic analysis. Further, I withdrew his L-dopa with no adverse consequences.

Whilst this all appeared reasonable to me, not to say appropriate, the patient took the loss of his diagnostic label very badly. Although he returned for follow-up consultations, apparently interested in my endeavours, I learned from one of his children that he had become withdrawn, hardly talked to his family and certainly would 


\section{(before replacing)}

not discuss his illness. His general practitioner felt he had become clinically depressed and was considering antidepressants. When I broached the subject with the patient, he admitted being upset by my changing his diagnosis. He had felt secure with the name Parkinson's disease, understanding what it involved and, perhaps most importantly, that other people had heard of this disorder. The loss of this 'label' of Parkinson's disease meant a return of insecurity. Not being given a recognisable replacement diagnosis meant that it was no longer possible for him simply to present others with a name. Moreover, that I had embarked on a new round of investigations meant he had to explain that I, and therefore he, was unsure exactly what was wrong.

I have no diagnostic denouement to give in this case, the man died of an unrelated cancer and my investigations failed to identify a cause of his neurological symptoms. These included the available genetic analyses for spinocerebellar atrophy types 1,2,3, dentato-rubro-pallidoluysian atrophy and Huntington's disease

My reason for bringing this case to the attention of others is to demonstrate that we may fail despite our best intentions. Whilst my management appeared appropriate to me, I had clearly worsened this patient's condition, or at least made it less bearable.

When one considers the question of a diagnosis, or rather a diagnostic label, I have always been aware of the negative aspects associated with labelling. One learns of the stigma attached to many labels, particularly psychiatric ones. For example, in the current Royal College of Psychiatrists 'Changing minds' campaign information I found the following: 'Diagnosis runs the risk of attaching an enduring 'label' to a person, which can become the focus of distorted negative images in the media and in the public mind. These images can then lead to further discrimination...'

Nevertheless, having a diagnosis has clear advantages, not just from the viewpoint of the medical profession. A label is a form of shorthand enabling one person to transmit meaning without lengthy description. For the patient, it means many things, not least the recognition that they suffer (something perhaps that drives the debate surrounding the chronic fatigue syndrome). In social terms, a diagnosis that most people recognise can mean that one avoids the need for lengthy explanation, something that often serves as a reminder of the state of being sick. Whatever the benefit, patients do become attached to their label.

How common is this problem of removing a label and making the patient worse? I have had at least one other similar experience, again with the diagnostic label of Parkinson's disease, where I changed it to corticobasal degeneration. Not only is this a longer title, but it also contains words not easily understood and the term degeneration, which from the lay perspective sounds rather unpleasant. As someone who tends toward highly specialised areas such as neurogenetics and mitochondrial disease, I am forced to ask myself whether it is just me that has this problem? If so, I am exposed to the ridicule I feared when starting this article. I suspect that this is not the case and I hope that others will recognise similar patients in their own clinical practice.

Because my experience suggests that the harm caused by re-labelling is not rare, what should one do when replacing a recognised diagnosis with one that perhaps we understand, but that the patient does not? Here, I am afraid, I do not have an easy answer, only a comment. I believe strongly that we should be honest with our patients, thus investigating someone without informing them of the reason would be difficult. There is, however, the element of timing, and perhaps my mistake was over zealousness. I certainly think more carefully now before replacing a diagnostic label, particularly Parkinson's disease.

Is this a problem of the past? The easy access to medical information through the Internet means that patients today are more knowledgeable and more cognizant of the breadth of diagnostic possibilities. Not everyone feels capable of tackling the computer age however, and common amongst these will be individuals at risk of developing late onset disorders such as Parkinson's disease. Lastly, one could ask whether this is something peculiar to the diagnosis of Parkinson's disease? I think not. The diagnosis of multiple sclerosis seems to me to offer similar scope for overzealous relabelling. For me, these difficulties are a pertinent reminder of the human element that pervades medicine and probably what keeps me in clinical medicine and not just in the (relative) security of my laboratory.

\section{I certainly think}

more carefully now

before replacing

a diagnostic

label, particularly

Parkinson's disease 